\title{
Influence des enzymes coagulantes et de la plasmine sur la croissance des levains en début de fabrication de l'emmental. Étude préliminaire sur milieux modèles
}

\author{
A Baer, I Ryba, M Casey \\ Station Fédérale de Recherches laitières, CH-3097 Liebefeld, Suisse
}

\begin{abstract}
Résumé - Les conditions de croissance de $S$ thermophilus et de L delbrueckii subsp lactis dans l'emmental sont mal connues ; ces bactéries disposent d'un système protéinasique peu important, et leur croissance pourrait dépendre de la protéolyse primaire des caséines par les enzymes coagulantes et la plasmine. Les résultats présentés, obtenus lors de la simulation des 24 ou 36 premières heures de fabrication d'emmental, ont montré que : 1) la caséine $\alpha_{\mathrm{s} 1^{-I}}$, issue de l'hydrolyse de la caséine $\alpha_{\mathrm{s} 1}$ par la présure, apparaît dans le lactosérum dès le décaillage ; sa concentration augmente durant l'affinage, démontrant une activité protéolytique résiduelle de la présure dans l'emmental, malgré le chauffage du fromage à $53^{\circ} \mathrm{C} ; 2$ ) la croissance des bactéries ne dépend pas de la concentration en acides aminés libérés par l'activité protéolytique des bactéries ; 3) l'accumulation des acides aminés et la croissance des lactobacilles augmentent parallèlement lorsqu'on ajoute quatre fois plus de présure ; il n'y a pas d'influence sur les streptocoques ; 4) les lactobacilles se développent sur les caséines dans l'ordre préférentiel : $\beta<\alpha_{\mathrm{s} 1}+$ présure $>\alpha_{\mathrm{s} 1}$. Il n'y a pas de croissance sur la caséine $\kappa$ même après hydrolyse par la présure ; 5) une concentration de plasmine quatre fois supérieure à la concentration moyenne du lait n'agit ni sur la croissance, ni sur l'accumulation des acides aminés en présence de la présure ou de $M$ miehei ; elle renforce l'action protéolytique de E parasitica. En revanche, un lait préhydrolysé par la plasmine, avant sa transformation en fromage, favorise la vitesse de croissance des lactobacilles.
\end{abstract}

streptocoques / lactobacilles / croissance / enzymes coagulantes / plasmine

Summary - Influence of coagulating enzymes and plasmin on the growth of starter bacteria at the start of Emmental cheese manufacture. Preliminary report. The growth conditions of $S$ thermophilus and $L$ delbrueckii subsp lactis in Swiss-type cheese have not been fully studied.

Communication orale présentée au symposium 'Ripening and Quality of Cheeses', Besançon, France, 26-28 février 1996. 
Since these bacteria show a low proteinase activity, it was assumed that their growth in cheese could depend on the preliminary hydrolysis of caseins by coagulating enzymes and plasmin. The purpose of this work was to develop a simple method which would allow us to measure the effect of casein hydrolysates produced by the coagulating enzymes of rennet, E parasitica and $M$ miehei, as well as by plasmin, on the growth of starter bacteria during simulated cheese manufacture. The growth of one starter used for Emmental manufacture was studied in defatted UHT milk, containing calcium carbonate to maintain a constant $\mathrm{pH}$ similar to that of cheese. The various steps (temperature, duration) of cheese manufacture for 24 or $36 \mathrm{~h}$ were then simulated; samples were taken at various times for analysis. Proteolysis of caseins was assessed by the quantitative reaction of free amino acids with a cadmium-ninhydrin reagent. In order to study the specific influence of each of the casein types on the growth of bacteria, a growth medium was developed by 'exhaustion' of the protein fraction from a normal medium. The kinetics of the bacterial growth were followed photometrically. The results showed that: 1) $\alpha_{\mathrm{s1}}$-I casein, produced by the proteolytic activity of rennet, was already present in whey after curd formation; its concentration increased during ripening, thus demonstrating the presence of residual proteolytic activity of rennet in Emmental, in spite of the high cooking temperature; 2 ) the growth of bacteria did not depend on the amount of amino acids; 3 ) the production of free amino acids as well as the growth of lactobacilli increased at the same rate when four times more rennet was added to the milk; no such influence was observed with streptococci; 4) the growth of lactobacilli on caseins was shown to follow the following preferential order: $\beta$-casein $>\alpha_{\mathrm{s} 1}$-casein + rennet $>$ $\alpha_{s 1}$-casein; no growth could be observed on $\kappa$-casein, even after hydrolysis by rennet; 5 ) the addition of four times more plasmin than the average concentration encountered in milk did not increase either the liberation of amino acids or the growth of bacteria in the presence of rennet or $M$ miehei, but in the presence of $E$ parasitica enforced the proteolytic activity by the bacteria. Nevertheless, an increase in the rate of bacterial growth could be observed when the milk was pre-incubated with plasmin before its transformation to cheese.

\section{streptococci / lactobacilli / growth / coagulating enzymes / plasmin}

\section{INTRODUCTION}

Le développement du goût et de la texture des fromages durant leur affinage est principalement dû à la dégradation enzymatique des caséines par les protéinases et peptidases des bactéries lactiques des levains et, dans une moindre mesure, des germes contaminants du lait. Par conséquent, l'intensité de la croissance des bactéries en début de fabrication influencera directement le potentiel protéolytique présent dans le fromage. La croissance des bactéries lactiques nécessite des peptides et des acides aminés, dont la concentration est très faible dans le lait cru de bonne qualité (Reiter et Oram, 1962). Ces bactéries doivent donc disposer d'un système protéolytique important qui leur permette d'utiliser les protéines du lait comme source d'azote ; ce système est peu développé chez Lactobacillus delbrueckii subsp lactis utilisé dans la fabrication au lait cru des fromages helvétiques à pâte dure (Hunter, 1950 ; Meyer et al, 1989 ; Sasaki et al, 1995), dont la croissance est plus lente dans le lait que dans un milieu de culture. Par conséquent, on peut admettre que la croissance de ces germes dans les fromages dépend de la conversion primaire des caséines en peptides par d'autres protéinases (enzymes coagulantes, plasmine) et plus particulièrement de la formation, par la présure, de la caséine $\alpha_{\mathrm{sI}}$-I et de son peptide $\alpha_{\mathrm{s} 1}$-CN (f1-23) (Exterkate, 1987), substrats dégradés en acides aminés par les pepti- 
dases des levains (Visser, 1977). Toutefois, tous les travaux qui démontrent l'influence de la présure sur la protéolyse des caséines par les bactéries lactiques ont été effectués sur des fromages à pâte mi-dure (gouda, cheddar), dont la température de fabrication, inférieure à $45^{\circ} \mathrm{C}$, ne dénature pas la chymosine, alors que dans l'emmental, chauffé à $53{ }^{\circ} \mathrm{C}$, on ne retrouve que $1 \%$ de l'activité coagulante ajoutée (Garnot et Mollé, 1987) ou même plus d'activité du tout (Matheson, 1981 ; Casey, obs pers). Toutefois, Collin et al (1987) ont démontré que la caséine $\alpha_{\mathrm{s1}}$-I formait les $4 \%$ des caséines totales dans le gruyère de Comté, chauffé à $53,5^{\circ} \mathrm{C}, 20$ heures déjà après fabrication ; et nous avons aussi rapporté une observation semblable dans l'emmental, suggérant que l'action de la chymosine pouvait produire la caséine $\alpha_{\mathrm{s}}$-I avant que la température de cuisson n' atteigne $53{ }^{\circ} \mathrm{C}$ (Baer, 1995).

La plasmine, protéinase alcaline native du lait, est une autre enzyme dont l'activité protéolytique libère des peptides susceptibles d'être convertis en oligopeptides et acides aminés par les enzymes des bactéries lactiques durant l'affinage des fromages (Visser, 1977 ; Miranda et Gripon, 1986 ; Collin et al, 1987 ; Fox, 1989) et qui semble aussi jouer un rôle important dans la protéolyse primaire des caséines dans le fromage de gruyère (Casey et al, 1987).

Ces remarques préliminaires montrent que les conditions de croissance des bactéries lactiques dans les fromages à pâte dure sont mal connues. Dans ce travail, nous avons étudié l'influence de la protéolyse primaire des caséines par les enzymes coagulantes microbiennes d'Endothia parasitica (récemment dénommée Cryphonectria parasitica, mais l'ancienne dénomination a été conservée dans le texte), de Rhizomucor miehei, de la présure (chymosine) et de la plasmine, sur la protéolyse tertiaire des caséines (libération des acides aminés) par les bactéries lactiques, ainsi que sur leur croissance. Lactobacillus delbrueckii subsp lactis, utilisé dans la fabrication des emmentals helvétiques, a été plus particulièrement étudié.

\section{MATÉRIEL ET MÉTHODES}

\section{Matériel}

\section{Réactifs}

Tous les produits chimiques étaient de qualité analytique. La plasmine et les caséines $\alpha_{\mathrm{sl}}, \beta$ et $\kappa$ provenaient de chez Sigma (Buchs, Suisse). Les enzymes coagulantes liquides, présure de veau standard, Rennilase ( $R$ miehei) et Suparen ( $E$ parasitica) ont été fournies par Winkler (Konolfingen, Suisse). L'extrait de levures et le peptone «Trypticase» utilisés dans les milieux de culture provenaient de chez Difco (Chemie Brunschwig, Bâle, Suisse).

\section{Bactéries}

Le levain, culture mixte, utilisé pour la fabrication de l'emmental, a été fourni par notre département de microbiologie. Les souches de $L \mathrm{del}$ brueckii subsp lactis ont été isolées du levain par notre section de biochimie.

\section{Méthodes}

\section{Croissance des bactéries \\ dans un milieu de culture}

Le milieu LS-5 utilisé pour la croissance des souches de lactobacilles a été développé dans notre département (Casey et Meyer, 1985). C'est un milieu semblable au MRS, mais mieux tamponné.

Pour étudier l'influence spécifique des caséines sur la croissance des souches isolées. un milieu minimum a été préparé par «épuisement» ; le milieu LS-5, dépourvu de peptone mais contenant l'extrait de levures, a été ensemencé avec les lactobacilles. Après croissance, moins intensive que dans le milieu complet, les bactéries ont été éliminées par centrifugation stérile et le pH réajusté à 6,5 . Après chauffage pendant 25 minutes à $80^{\circ} \mathrm{C}$ pour détruire toute 
activité enzymatique, $2,0 \mathrm{~mL}$ de lactose $0,5 \mathrm{~mol} / \mathrm{L}$ ont été rajoutés à $100 \mathrm{~mL}$ du milieu.

L'influence des caséines ou de leurs hydrolysats sur la croissance des bactéries a été étudiée en ajoutant $200 \mu \mathrm{L}$ de la solution de protéines $(15 \mathrm{~g} / \mathrm{L})$ à $10 \mathrm{~mL}$ du milieu épuisé. Les lactobacilles, cultivés en milieu LS- 5 complet, ont été lavés deux fois dans une solution saline. Le volume de la suspension à ajouter à $10 \mathrm{~mL}$ du milieu épuisé pour obtenir une densité optique de 0,008 , mesurée à $650 \mathrm{~nm}$, a été déterminé et ajouté au milieu épuisé. La quantité totale de germes ajoutée à $10 \mathrm{~mL}$ correspondait à environ $210^{6}$. La croissance des bactéries a été suivie par mesure photométrique à $650 \mathrm{~nm}$.

\section{Croissance des bactéries dans le lait}

L'étude de la croissance des bactéries du levain dans le lait a été réalisée dans $40 \mathrm{~mL}$ de lait maigre UHT, contenant $1,25 \mathrm{~g}$ de carbonate de calcium pour maintenir le $\mathrm{pH}$ à une valeur d'environ 5,5 , similaire à celle du fromage, soumis aux conditions (température, durée) simulées de la fabrication d'Emmental, comme déjà décrit (Baer, 1995). Après les 24 premières heures de fabrication simulée, les échantillons ont été déposés, sous agitation constante, dans une cave froide $\left(14^{\circ} \mathrm{C}\right)$, durant 17 jours, puis transférés dans une cave d'affinage $\left(22^{\circ} \mathrm{C}\right)$ jusqu' au $28^{\mathrm{e}}$ ou $55^{e}$ jour. L'intensité de la protéolyse a été déterminée par l'analyse des acides aminés libérés, selon la méthode sur plaque de microtitration décrite ailleurs (Baer et al, 1996).

\section{Électrophorèse en gel de polyacrylamide (PAGE)}

L'analyse électrophorétique de la caséine $\alpha_{\mathrm{s} 1^{-I}}$ a été réalisée de la façon suivante : les échantillons des laits, soit de chaudière, soit de fabrication simulée ont été prélevés à des temps différents de fabrication, dès le décaillage. Après avoir laissé sédimenter le caillé, à $4{ }^{\circ} \mathrm{C}$, durant $1 \mathrm{~h}$, le surnageant a été prélevé et son $\mathrm{pH}$ abaissé à 4,6 . Après centrifugation (tubes Eppendorf) pendant $10 \mathrm{~min}$ à $16000 \mathrm{~g}$, le culot a été repris dans de l'urée $10 \mathrm{~mol} / \mathrm{L}$, contenant $4 \mathrm{~mol} / \mathrm{L}$ de dithiothreitol. L'électrophorèse a été effectuée avec un appareil Midget-LKB (Pharmacia, Dübendorf, Suisse) sur minigel de polyacrylamide, $12 \%$, contenant $4 \mathrm{~mol} / \mathrm{L}$ d'urée, comme décrit par de Jong (1975).

\section{RÉSULTATS}

\section{Hydrolyse de la caséine $\alpha_{s I}$ en $\alpha_{3} I^{-I}$ par les enzymes coagulantes}

La figure 1 montre l'influence de la température de chauffage sur l'apparition de la caséine $\alpha_{s 1}$-I après un, dix et 40 jours d'affinage dans trois fromages de types différents, fabriqués avec la même présure. On constate que dans le fromage à raclette, chauffé à $42^{\circ} \mathrm{C}$, la bande caractéristique apparaît nettement, dès le premier jour (piste 1) et que l'intensité de sa coloration va en s'intensifiant durant l'affinage, accompagnée, après 40 jours, d'une nette diminution de la bande correspondant à la caséine $\alpha_{\mathrm{s} 1}$. Ce phénomène s'observe également dans les électrophorégrammes des deux autres fromages, mais s'atténue en fonction de la température de chauffage : la caséine $\alpha_{\mathrm{s} 1}$-I est peu visible dans l'emmental, chauffé à $53{ }^{\circ} \mathrm{C}$, après 1 jour (piste 4) et n'apparait qu'en trace dans le gruyère fabriqué à $56^{\circ} \mathrm{C}$ (piste 7). Après 40 jours, la bande caractéristique est bien présente dans l'électrophorégramme de l'emmental, alors qu'elle est moins visible dans le gruyère.

Ces résultats démontrent que, malgré la température élevée de chauffage des fromages à pâte dure, une activité protéolytique résiduelle de la présure est présente durant la période d'affinage. Mais pour que la caséine $\alpha_{\mathrm{s1}^{-}}$I ou le peptide libéré $\alpha_{\mathrm{s} 1}-\mathrm{CN}$ (f1-23) puissent éventuellement servir de source d'azote à la croissance des bactéries lactiques, il faut qu'ils soient présents dans le lait dès les premières heures de fabrication. Des analyses électrophorétiques réalisées avec le lait prélevé dans la chaudière durant la fabrication de plusieurs emmentals dans notre fromagerie expérimentale ont montré 
l'apparition de la caséine $\alpha_{\mathrm{s1}}$-I dans le lactosérum, dès la fin du décaillage (fig 2).

L'influence des enzymes coagulantes de $R$ miehei (MM) et d'E parasitica (EP) sur l'apparition de la caséine $\alpha_{\mathrm{s} 1}-\mathrm{I}$ dans le lait après les premières 24 heures de fabrication simulée a aussi été étudiée. Des analyses électrophorétiques ont montré que la caséine $\alpha_{s_{1}}-\mathrm{I}$ apparaît clairement en présence de présure et de MM, alors que l'étalement de la bande de la caséine $\alpha_{s 1}$ après coagulation du lait par EP ne permet pas d'identifier avec certitude la bande de la caséine $\alpha_{\mathrm{s} 1^{-}}$I (résultats pas montrés). En répétant cette expérience avec une solution de caséine $\alpha_{\mathrm{s}}$ isolée, à la place de lait et en procédant à une électrophorèse en présence de SDS, on a remarqué que la conversion en caséine $\alpha_{\mathrm{s} 1^{-}}$I par MM est presque totale, alors que la présure n'a hydro-

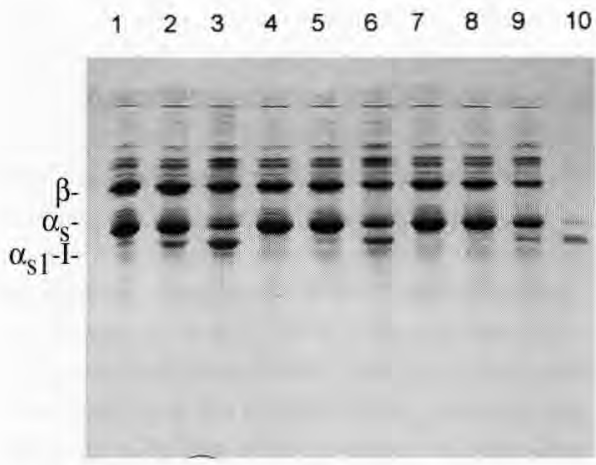

Fig 1. Profils des caséines obtenus en PAGE avec urée, de trois types de fromages, en fonction du temps d'affinage. Pistes 1, 2, 3: Raclette après 1,10 et 40 jours d'affinage respectivement ; pistes 4, 5, 6 : Emmental après 1,10 et 40 jours ; pistes $7,8,9$ : Gruyère après 1,10 et 40 jours ; piste 10 : caséine $\alpha_{\mathrm{s} 1}$ après hydrolyse par la présure. La bande anodique foncée correspond à $\alpha_{\mathrm{s} 1}-\mathrm{I}$.

Urea-PAGE patterns of three various cheeses as a function of time of ripening. Lanes 1, 2, 3: Raclette after 1, 10 and 40 d of ripening; lanes 4, 5, 6: Emmental after 1,10 and $40 \mathrm{~d}$; lanes 7, 8, 9: Gruyère after 1 , 10 and 40 d; lane 10: $\alpha_{\mathrm{S1}}$-casein after hydrolysis by rennet. The anodic dark band correspond to $\alpha_{\mathrm{s} 1}-1$ casein. lysé qu'environ la moitié de la caséine native. En présence de EP, la bande qui devrait correspondre à la caséine $\alpha_{\mathrm{s}}$-I a migré légèrement plus lentement qu'avec les autres enzymes, et l'électrophorèse a aussi montré un dédoublement de cette bande. Ces résultats expliquent l'aspect étalé de la région $\alpha_{\mathrm{s}}$ et indiquent que les produits de dégradation de la caséine $\alpha_{\mathrm{s}}$ par EP pourraient être de poids moléculaire supérieur à celui de la caséine $\alpha_{\mathrm{s1}}$-I ; le peptide libéré contiendrait alors moins d'acides aminés que la caséine $\alpha_{\mathrm{s} 1}-\mathrm{CN}(\mathrm{f} 1-23)$.

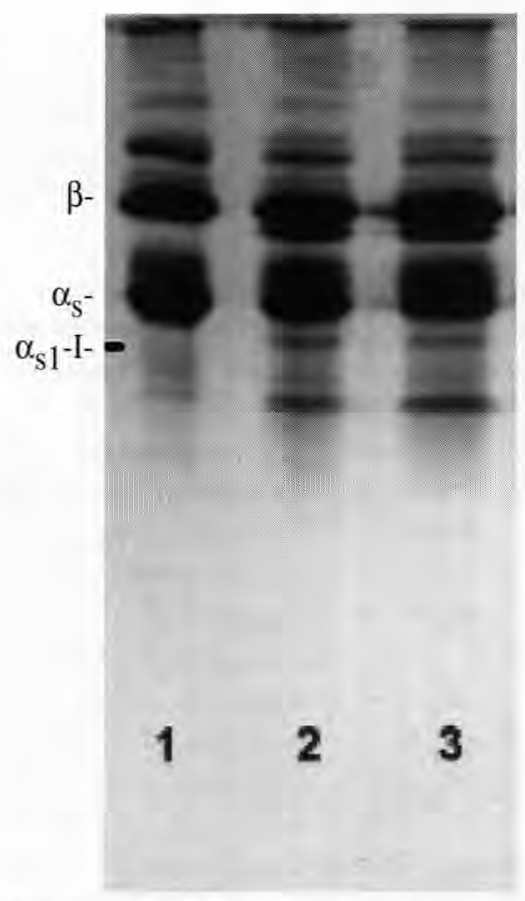

Fig 2. Profils des protéines, obtenus en PAGE avec urée, du petit lait de chaudière, prélevé en cours de fabrication. Piste 1 : lait avant l'emprésurage ; pistes 2,3 : petits laits prélevés après le décaillage et en fin de chauffage à $53{ }^{\circ} \mathrm{C}$, respectivement.

Urea-PAGE patterns of whey proteins taken from the vat at different times of manufacture. Lane 1: milk before addition of rennet; lanes 2, 3; wheys sampled after cutting the curd and after heating to $53{ }^{\circ} \mathrm{C}$ respectively. 
Tableau I. Influence des enzymes coagulantes sur l'accumulation des acides aminés par un levain mixte (CM), mesurée du $1^{\mathrm{er}}$ au $28^{\mathrm{e}}$ jour d'une fabrication simulée d'emmental.

Influence of coagulating enzymes on the accumulation of amino acids by a mixed starter (CM) as measured between the 1 st and the 28 th day of a simulated Emmental manufacture.

Ajouts au lait UHT

Durée de simulation (j)

\begin{tabular}{llrrr} 
& 1 & 2 & 17 & 28 \\
& $a a^{\mathrm{a}}$ & $a a$ & $a a$ & $a a$ \\
\hline Présure & 1,7 & 1,8 & 1,8 & 1,9 \\
$\mathrm{CM}$ & $1,7 \mathrm{~b}$ & 3,2 & 7,3 & 14,4 \\
$\mathrm{CM}+$ présure $^{\mathrm{b}}$ & 6,2 & 8,8 & 17,2 & 29,6 \\
$\mathrm{CM}+$ miehe $^{\mathrm{c}}$ & 5,8 & 7,6 & 26,0 & 25,3 \\
$\mathrm{CM}+$ parasitica $^{\text {d }}$ & 2,1 & 4,9 & 8,2 & 13,1 \\
\hline
\end{tabular}

a Acides aminés libérés; mmol/L équivalent leucine. b Les valeurs des acides aminés du lait seul qui augmentent de 1,8 à 2,2 au $28^{\mathrm{e}}$ jour ont été soustraites des mesures présentées. ${ }^{\mathrm{C}}$ Rennilase ${ }^{\circledR}$. d Suparen ${ }^{\circledR}$.

${ }^{a}$ Liberated amino acids; mmol/L leucin equivalent. ${ }^{b}$ Values of amino acids in milk alone which increased from 1.8 to 2.2 on the 28 th $d$ were substracted from the values shown. ${ }^{c}$ Rennilase ${ }^{\circledR}$. $d$ Suparen ${ }^{\circledR}$.

\section{Influence des enzymes coagulantes} sur la croissance des bactéries lactiques d'un levain et l'accumulation des acides aminés

Le tableau I montre que l'accumulation des acides aminés augmente régulièrement durant toute la durée de la simulation des 28 premiers jours de maturation, y compris durant la période en cave froide à $14^{\circ} \mathrm{C}$, du premier au $17^{\mathrm{e}}$ jour. La présure et l'enzyme coagulante de MM provoquent, dès le premier jour, une accumulation des acides aminés environ quatre fois supérieure à celle mesurée dans le lait sans enzyme coagulante. Cette différence subsiste tout au long de l'expérience. En revanche, la coagulation du lait par EP ne provoque pas d'augmentation notable de la concentration en acides aminés, ce qui pourrait s'expliquer par la présence d'un substrat différent de ceux obtenus avec les deux autres enzymes coagulantes, comme décrit cidessus. L'accumulation des acides aminés par les bactéries est donc augmentée par la présure ou MM, ce qui confirme les résultats de Visser (1977).
Le rôle de l'hydrolyse de la caséine $\alpha_{\mathrm{s} 1}$ par la présure sur la croissance et la protéolyse tertiaire des caséines par les bactéries lactiques a été étudié en ajoutant quatre fois plus d'enzyme au lait. Des souches de streptocoques thermophiles et de lactobacilles ont été analysées séparément durant les 36 premières heures de fabrication simulée dans le lait. Les résultats (tableau II) montrent qu'après 24 heures, l'accumulation des acides aminés par les streptocoques n'est pas modifiée par la présure, car leur concentration, très basse, correspond à celle d'un lait UHT seul. Après 36 heures, la concentration en acides aminés n'a pas changé. Cela pourrait indiquer que le système protéolytique des streptocoques thermophiles ne reconnaît pas les caséines $\alpha_{\mathrm{s} 1}-\mathrm{I}$ et $\alpha_{\mathrm{s} 1^{-}} \mathrm{CN}(\mathrm{f} 1-23)$ comme substrats, ou que leurs peptidases n'ont pas encore été libérées dans le milieu par la lyse cellulaire (Meyer, comm pers).

L'accumulation des acides aminés, en présence de quatre fois plus de présure, a augmenté d'environ $50 \%$ après 24 heures. Après 36 heures, l'augmentation des acides aminés n'est plus que de $20 \%$. Ces résultats montrent 
Tableau II. Influence de la présure sur l'accumulation des acides aminés par $L$ delbrueckii subsp lactis et $S$ thermophilus dans le lait, mesurée après 24 et 36 heures de fabrication simulée d'emmental.

Influence of rennet on the accumulation of amino acids by $\mathrm{L}$ delbrueckii subsp lactis and $\mathrm{S}$ thermophilus as measured in milk after 24 and 36 h of simulated Emmental manufacture.

Durée de simulation $(h)$

$24 \quad 36$

$a a^{\text {a }} \quad a a$

$\begin{array}{lll}\text { L lactis } & 1,2 \mathrm{~b} & 3,1 \\ \text { L lactis + présure } & 3,4 & 4,8 \\ \text { L lactis + présure } \times 4 & 5,2 & 5,8 \\ \text { S thermophilus } & 1,8 & 1,8 \\ \text { S thermophilus + présure } & 2,0 & 2,5 \\ \text { S thermophilus + présure } \times 4 & 2,2 & 0,7\end{array}$

\footnotetext{
a Acides aminés libérés, mmol/L équivalent leucine. ${ }^{b} \mathrm{La}$ valeur des acides aminés du lait seul, de 2,3 , a été soustraite des trois mesures avec $L$ lactis.

${ }^{a}$ Liberated amino acids, mmol/L leucin equivalent. ${ }^{b}$ The value of 2,3 of amino acids in milk alone was substracted from the 3 values measured with $\mathrm{L}$ lactis.
}

que l'hydrolyse de la caséine $\alpha_{\mathrm{s} 1}$ par la présure favorise l'accumulation des acides aminés en présence des lactobacilles. Ils suggèrent aussi que la présure pourraît être le facteur limitant de la production initiale des acides aminés, comme proposé par Exterkate et Alting (1995). Toutefois, des résultats récents obtenus après soumission du manuscript ont montré que l'accumulation des acides aminés était proportionnelle à l'intensité de la lyse des lactobacilles ; les peptidases ainsi libérées dans le milieu dégradent alors les peptides en acides aminés.

La présence de la caséine $\alpha_{\mathrm{s} 1}$-I dans le caillé d'Emmental déjà avant la cuisson et l'augmentation de la protéolyse tertiaire par les lactobacilles en présence de présure lors de la fabrication simulée indiquent que les caséines $\alpha_{\mathrm{s1}}$-I et $\alpha_{\mathrm{s} 1}-\mathrm{CN}$ (f1-23) pourraient servir de substrats au système protéolytique de ces bactéries, comme démontré pour Lactococcus lactis subsp cremoris par Exterkate et Alting (1992), et servir de source d'azote à la croissance des germes. Cette hypothèse a été vérifiée en étudiant la cinétique de croissance d'une souche de $L$ del- brueckii subsp lactis dans $10 \mathrm{~mL}$ de milieu «épuisé», additionné de $200 \mu \mathrm{L}$ d'une solution de concentration identique $(15 \mathrm{~g} / \mathrm{L})$ de la caséine $\alpha_{\mathrm{sl}}, \beta$ ou $\kappa$, soumise à l'hydrolyse de la présure durant les premières 24 heures de fabrication simulée. La figure 3 montre que la vitesse de croissance en présence de la caséine $\alpha_{\mathrm{s} 1}$ est très lente, mais qu'elle est doublée après son hydrolyse par la présure. Toutefois, elle est inférieure de moitié à la vitesse de croissance observée avec la caséine $\beta$ sur laquelle l'activité protéolytique de la présure n'a pas d'effet. Enfin, les lactobacilles sont incapables d'utiliser la caséine $\kappa$ comme source d'azote, ni ses hydrolysats, la para-K-caséine et le glycomacropeptide. Ces résultats suggèrent que, dans le lait, les lactobacilles utilisent préférentiellement, pour leur croissance, la caséine $\beta$ à la caséine $\alpha_{s 1}$ hydrolysée. On peut aussi évoquer une similitude entre le type de protéase dont disposeraient les souches de lactobacilles analysées et le type $\mathrm{P}_{1}$ décrit pour la souche HP de $L$ lactis subsp cremoris, dont la caséine $\beta$ est le substrat préférentiel, comparé aux caséines $\alpha_{\mathrm{s} 1}$ et $\kappa$ (Exterkate et De Veer Gerrie, 1987). 


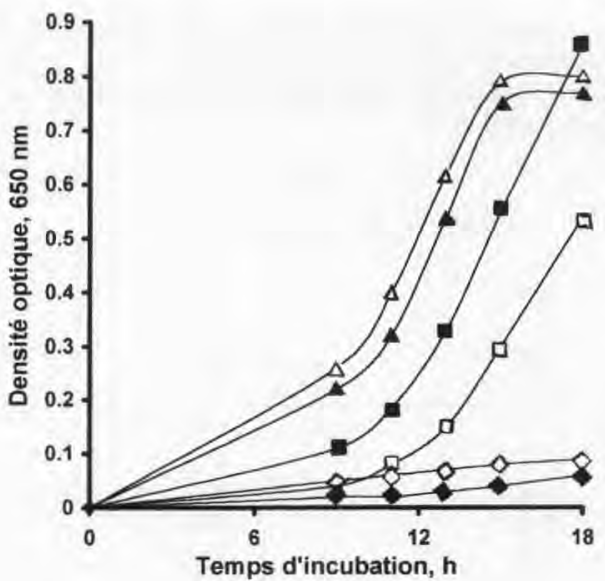

Fig 3. Courbes de croissance de $L$ delbrueckii subsp lactis dans $10 \mathrm{~mL}$ de milieu «épuisé», additionné de $200 \mu \mathrm{L}$ d'une solution de caséine $(15 \mathrm{~g} / \mathrm{L})$, soumise à 24 heures de fabrication simulée en présence ou non de présure. Milieu «épuisé» additionné des caséines $\alpha_{\mathrm{s}}$ sans $(\square)$, avec présure $(\mathbf{G})$; $\beta$ sans $(\boldsymbol{A})$, avec présure $(\Delta) ; \kappa$ sans $(\bullet)$, avec présure $(\Delta)$.

Growth of L delbrueckii subsp lactis in $10 \mathrm{~mL}$ of 'exhausted' medium, to which $200 \mu \mathrm{L}$ of a casein solution $(15 \mathrm{~g} / \mathrm{L})$ was added afier $24 \mathrm{~h}$ of simulated cheese manufacture with or without rennet. 'Exhausted' medium with caseins $\alpha_{s /}$ without $(\square)$, with rennet ( $\boldsymbol{E}$ ); $\beta$ without ( $\mathbf{\Delta})$, with rennet $(\Delta) ; \kappa$ without (-), with rennet $(\diamond)$

\section{Influence de la plasmine sur l'accumulation des acides aminés et la croissance des bactéries lactiques d'un levain}

L'ajout au lait UHT, dépourvu de toute activité résiduelle de l'enzyme, de $0,35 \mathrm{mg} / \mathrm{L}$ de plasmine, concentration moyenne du lait (Richardson et Pearce, 1981) n'a pas provoqué de modification notable de l'accumulation des acides aminés, même après $4 \mathrm{j}$ d'incubation des laits avec le levain à $30^{\circ} \mathrm{C}$. Pour cette raison, les résultats présentés ici ont été obtenus après adjonction de $1,5 \mathrm{mg} / \mathrm{L}$ de plasmine.

Le tableau III montre que l'enzyme n'a aucun effet sur l'accumulation des acides aminés dans les laits sans enzymes coagulantes, ni dans les échantillons coagulés par la présure ou par MM.
Une prolongation de l'expérience jusqu'au $55^{\mathrm{e}}$ jour n'a pas modifié significativement les valeurs du tableau III. Ces résultats confirment ceux qui ont déjà été publiés (Baer, 1995); ils indiquent que les peptides produits par la présure ou par MM ne sont pas hydrolysés par la plasmine ou, s'ils le sont, les produits obtenus ne sont pas dégradables en acides aminés par l'activité protéolytique des bactéries du levain, tout comme les peptides, directement issus de l'activité de l'enzyme.

En revanche, on trouve environ 1,5 fois plus d'acides aminés libres lorsque la plasmine est ajoutée en présence de EP. Cette différence reste constante jusqu'à la fin du stockage en cave froide. Dès que les échantillons sont soumis à la température plus élevée de la cave d'affinage, l'accumulation des acides aminés augmente régulièrement. Après $55 \mathrm{j}$, elle est 2,2 fois plus élevée (ce dernier résultat ne figure pas au tableau III). Ces résultats suggèrent que l'activité protéolytique de EP a libéré d'autres peptides que la caséine $\alpha_{\mathrm{s} 1}$-I et $\alpha_{\mathrm{s} 1}$-CN (f1-23) qui sont, à leur tour, hydrolysés par la plasmine, puis par les peptidases des bactéries lactiques en acides aminés. L'accumulation des peptides a vraisemblablement eu lieu en tout début de la fabrication simulée, car EP est encore plus sensible à la thermodénaturation que la chymosine de la présure (Garnot, 1985).

La vitesse de croissance des lactobacilles du levain est augmentée lorsque du lait, soumis à l'hydrolyse de 1,5 ou $3,0 \mathrm{mg} / \mathrm{L}$ de plasmine durant 3 jours à $30^{\circ} \mathrm{C}$, est utilisé comme source d'azote dans le milieu «épuisé». La figure 4 montre que les vitesses de croissance des bactéries sur le lait seul ou additionné de $1,5 \mathrm{mg} / \mathrm{L}$ de plasmine sont très similaires, lorsque le lait n'est incubé que durant les 24 premières heures de fabrication simulée. En revanche, le lait préalablement incubé avec la plasmine pendant 3 jours augmente la vitesse de croissance, d'autant plus que la concentration de l'enzyme est grande. Ces résultats indiquent que le temps nécessaire à l'enzyme pour hydrolyser les caséines est relativement long, même à une concentration quatre fois supérieure à la concentration moyenne du 
Tableau III. Influence de la plasmine sur l'accumulation des acides aminés par un levain mixte (CM), mesurée $\mathrm{du} 1^{\mathrm{er}}$ au $28^{\mathrm{e}}$ jour d'une fabrication simulée d'emmental.

Influence of plasmin on the accumulation of amino acids by a mixed starter (CM) as measured between the 1st and the 28th day of a simulated Emmental manufacture.

Ajouts au lait UHT

Durée de simulation (j)

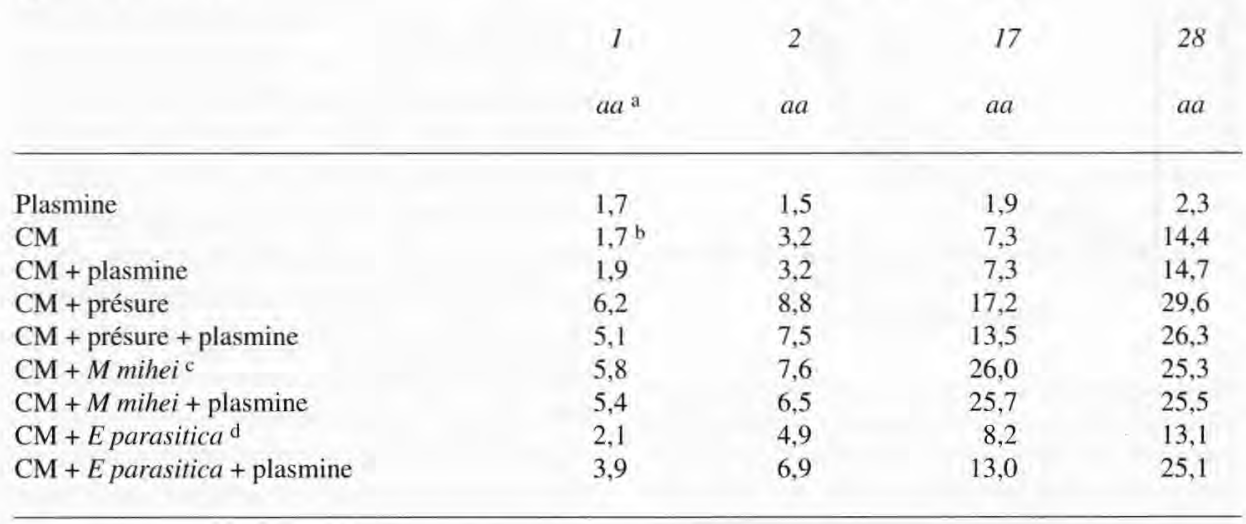

${ }^{a}$ Acides aminés libérés, mmol/L équivalent leucine. ${ }^{b}$ Les valeurs des acides aminés du lait seul qui augmentent de 1,8 à 2,2 au 28 c jour ont été soustraites des mesures présentées. " Rennilase ${ }^{\circledR}$. d Suparen ${ }^{\circledR}$.

${ }^{a}$ Liberated amino acids; mmol/L leucin equivalent. ${ }^{b}$ Values of amino acids in milk alone which increased from 1.8 to 2.2 on the 28 th $d$ were substracted from the values shown. ${ }^{c}$ Rennilase ${ }^{\circledR}$, ${ }^{d}$ Suparen ${ }^{\circledR}$.

lait, et que les peptides produits sont utilisés par les lactobacilles du levain pour leur croissance.

Il semble aussi que la concentration en plasmine native du lait soit insuffisante pour influencer la croissance des lactobacilles ou l'accumulation des acides aminés durant les 55 premiers jours d'affinage de l'emmental. Cela confirme les résultats d'une étude effectuée durant une année entière, sur le rapport entre la variation du taux de plasmine dans le lait de chaudière et la concentration de la fraction azotée soluble (WSN) dans les emmentals : malgré l'augmentation de l'activité de la plasmine dans les laits des mois d'hiver, due à la période de lactation après vêlage, aucune corrélation vraiment significative entre l'activité de l'enzyme et la fraction WSN n'a été observée (Baer et Ryba, 1991).

Toutefois, Collin et al (1987) ont démontré que les caséines $\gamma$ issues de l'hydrolyse de la caséine $\beta$ par la plasmine, apparaissaient déjà après 20 heures de fabrication du gruyère et que leur concentration en cours d'affinage dépendait du taux de plasmine dans le lait, alors que Benslimane et al (1990) démontraient que les laits à faible concentration en caséines $\gamma$ contenaient peu de plasmine, mais ceux contenant une forte proportion des caséines $\gamma$ ne présentaient pas une grande activité de l'enzyme.

D'une façon générale, l'importance de la plasmine sur la protéolyse des fromages à pâte dure en cours d'affinage est peut-être surestimée, car l'activité de l'enzyme est très réduite au $\mathrm{pH}$ de 5,0 à 5,3 de l'emmental, et la transformation du plasminogène en plasmine, en cours de maturation, est ralentie par les inhibiteurs des activateurs du plasminogène, actifs dans le lait cru utilisé (Richardson, 1983a). En revanche, on ne peut pas exclure que le taux de plasmine dans le lait au moment de la traite (Fox, 1989), dont la valeur varie en fonction de la période de lactation (Richardson, 1983b) n'influence la teneur en caséines $\gamma$ et la concentration de la 


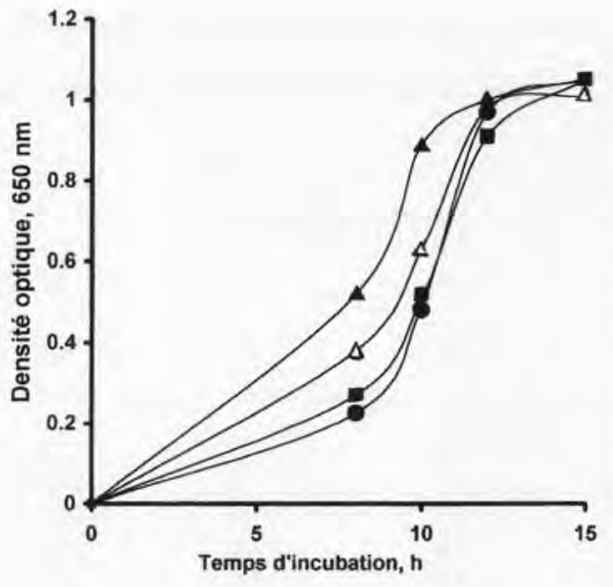

Fig 4. Courbes de croissance de $L$ delbrueckii subsp lactis dans $10 \mathrm{~mL}$ de milieu «épuisé», additionné de $200 \mu \mathrm{L}$ de lait UHT soumis à 24 heures de fabrication simulée en présence de plasmine, sans présure ni levain, ou préalablement incubé avec la plasmine durant 3 jours avant la simulation. Lait UHT sans plasmine (耳), avec quatre fois la concentration moyenne de plasmine dans le lait ( ) ; lait UHT incubé 3 jours avec quatre fois $(\Delta)$ et huit fois $(\boldsymbol{\Delta})$ respectivement la concentration moyenne de plasmine.

Growth of $\mathrm{L}$ delbrueckii subsp lactis in $10 \mathrm{~mL}$ of 'exhausted' medium, to which $200 \mu \mathrm{L}$ of UHT milk was added after its incubation for $24 h$ of simulated cheese manufacture with plasmin in the absence of rennet and starter, or previously incubated with plasmin for $3 d$ before simulation. UHT milk without plasmin ( $\boldsymbol{D})$, with four times the average concentration of plasmin in milk (-): UHT milk previously incubated for $3 d$ with four times $(\Delta)$ and eight times ( $\boldsymbol{\Delta}$ ) respectively the average plasmin concentration, before simulation.

fraction protéoses-peptones d'un lait, avant sa transformation en fromage, s'il n'est pas rapidement refroidi à la ferme. Il pourrait alors contenir suffisamment de peptides pour accélérer la vitesse de croissance des bactéries des levains lors de la fabrication des fromages ; cela pourrait être l'une des causes de l'augmentation de la concentration en acides aminés dans les Emmentals qui présentent une fermentation secondaire, surtout pour ceux fabriqués avec les laits d'hiver, dont la concentration en plasmine est élevée.

\section{CONCLUSION}

La méthode de fabrication simulée utilisée dans cette étude a permis d'obtenir rapidement et facilement des renseignements sur la croissance et le potentiel protéolytique des bactéries des levains ou de leurs souches isolées. En appliquant cette technique au criblage des levains de notre Station de recherches, il a été possible de choisir des bactéries et des levains en fonction de leur activité protéolytique et d'agir sur la concentration en acides aminés des emmentals, fabriqués dans notre fromagerie expérimentale (Baer, 1995). Les résultats préliminaires ont aussi montré que les caractéristiques protéolytiques d'un levain pouvaient dépendre de l'enzyme coagulante utilisée et que la croissance des lactobacilles ne dépendait pas de la concentration en acides aminés libres. Cela pourrait indiquer que l'accumulation des acides aminés est due aux peptidases des lactobacilles, échappées des cellules au moment de leur lyse. Dans ce cas, la lyse cellulaire devrait commencer dès le début de la croissance des bactéries.

L'application d'un milieu de culture «épuisé» à l'étude des substrats protéiques, préférentiellement dégradés par les bactéries lactiques, a permis de déterminer avec précision l'influence spécifique de chaque caséine sur la cinétique de croissance des germes. On pourrait utiliser cette méthode pour étudier la spécificité des systèmes protéolytiques et peptidasiques des bactéries en remplaçant les caséines par divers peptides.

\section{RÉFÉRENCES}

Baer A (1995) Influence of casein proteolysis by starter bacteria, rennet and plasmin on the growth of propionibacteria in Swiss-type cheese. Lait 75 , $391-400$

Baer A, Ryba I (1991) Influence de l'activité de la plasmine dans le lait sur la qualité du fromage d'emmental (fermentation secondaire). Rapp Int FAM 2/91

Baer A, Ryba I, Meyer J, Bütikofer U (1996) Microplate assay of free amino acids in Swiss cheeses. Food Sci Technol 29, 58-62 
Benslimane S, Dognin-Bergeret MJ, Berdagué JL, Gaudemer Y (1990) Variation with season and lactation of plasmin and plasminogen concentrations in Montbeliard cows' milk. J Dairy Res 57 , 423-435

Casey M, Gruskovnjak J, Fürst M (1987) Die Bedeutung von Plasmin bei der Reifung von Greyerzer Käse. Schweiz Milchw Forsch 16, 21-24

Casey M, Meyer J (1985) Presence of X-prolyl-dipeptidyl-peptidase in lactic acid bacteria. J Dairy Sci $68,3212-3215$

Collin JC, Berdagué JL, Dognin-Bergeret M, Grappin R (1987) Affinage et qualité du Gruyère de Comté. IV. Etude de la protéolyse. Lait 67, 299-318

de Jong L (1975) A quantitative electrophoretic method of studying cheese ripening. Neth Milk Dairy J 29, $162-168$

Exterkate FA (1987) On the possibility of accelerating the ripening of Gouda cheese: a comment. Neth Milk Dairy J 41, 189-194

Exterkate FA, De Veer Gerrie JCM (1987) Optimal growth of Streptococcus cremoris HP in milk is related to beta- and kappa-casein degradation. Appl Microbiol Biotechnol 25, 471-475

Exterkate FA, Alting AC (1992) The conversion of the $\alpha_{\mathrm{s1}}$-casein-(1-23) fragment by the free and bound form of the cell-envelope proteinase of $\mathrm{Lac}$ tococcus lactis subsp cremoris under conditions prevailing in cheese. System Appl Microbiol 16 , $1-18$

Exterkate FA, Alting AC (1995) The role of starter peptidases in the initial proteolytic events leading to amino acids in Gouda cheese. Int Dairy $J 5$, 15-28

Fox PF (1989) Proteolysis during cheese manufacture and ripening. J Dairy Sci 72, 1379-1400

Garnot P (1985) Heat stability of milk clotting enzymes. Technological consequences. Bull Int Dairy Fed 194, 2-7
Garnot P, Mollé D (1987) Heat stability of milk-clotting enzymes in conditions encountered in Swiss cheese making. J Food Sci 52, 75-77

Hunter GJE (1950) The growth requirement of lactobacilli in relation to cheese flavour development. J Dairy Res 17, 79-90

Matheson AR (1981) The immunological determination of chymosin activity in cheese. NZ J Dairy Sci Technol 15, 33-41

Meyer J, Howald D, Jordi R, Fürst M (1989) Location of proteolytic enzymes in Lactobacillus lactis and Streptococcus thermophilus and their influence on cheese ripening. Milchwissenschaft 44 , 678-681

Miranda G, Gripon, JC (1986) Origine, nature et incidences technologiques de la protéolyse dans le lait. Lait 66, 1-18

Reiter A, Oran JD (1962) Nutritional studies on cheeses starters. 1. Vitamin and amino acid requirements of single strain starters. J Dairy Res 29 , 63-77

Richardson B (1983a) Variation of the concentration of plasmin and plasminogen in bovine milk with lactation. N Z J Dairy Sci Technol 18, 247-252

Richardson B (1983b) The proteinases of bovine milk and the effect of pasteurization on their activity. NZ J Dairy Technol 18, 233-245

Richardson B, Pearce K (1981) The determination of plasmin in dairy products. NZ J Dairy Sci Technol 16, 209-220

Sasaki M, Bosman BW, Tan PST (1995) Comparison of proteolytic activities in various lactobacilli. J Dairy Res 62, 601-610

Visser FM (1977) Contribution of enzymes from rennet, starter bacteria and milk to proteolytic and flavour development in Gouda cheese. 3. Protein breakdown: analysis of the soluble nitrogen and amino acid nitrogen fractions. Neth Milk Dairy $J$ 31, 210-239 